\title{
Aspectos relacionados ao processo de retorno ao trabalho de indivíduos com desordens musculoesqueléticas do membro superior: uma bibliografia comentada*
}

\author{
Aspects connected with retorning to work process of \\ individuals with musculoesquelectical desorders: a \\ commented bibliography
}

\author{
Sandra Ribeiro da Silva ${ }^{1}$, Elaine Vieira Guimarães ${ }^{2}$, \\ Adriana Maria Valladão Novais Rodrigues ${ }^{3}$
}

SILVA, S. R.; GUIMARÃES, E. V.; RODRIGUES, A. M. V. N. Aspectos relacionados ao processo de retorno ao trabalho de indivíduos com desordens musculoesqueléticas do membro superior: uma bibliografia comentada. Rev. Ter. Ocup. Univ. São Paulo, v. 18, n. 1, p. 38-43, jan./abr., 2007.

\begin{abstract}
RESUMO: Retorno ao trabalho após um acidente/doença apresenta-se como um desafio para indivíduos, governos, médicos e reabilitadores, tornando-se alvo de preocupações constantes de pesquisadores. Pesquisas atuais têm destacado o aumento de desordens musculoesqueléticas no Membro Superior (MS) em todo o mundo. No Brasil, segundo estatísticas do INSS, 41,1\% dos acidentes registrados em 2003 atingiram o MS. O objetivo deste trabalho foi elaborar uma bibliografia comentada com análise criteriosa de artigos científicos identificando questões sobre a efetividade dos programas de retorno ao trabalho para indivíduos com desordens musculoesqueléticas em MS. Realizou-se consultas em base de dados eletrônicas entre 2000 e 2005. Com base nos critérios de inclusão, nove artigos foram selecionados e analisados pela qualidade das evidências, conforme a metodologia proposta por Law et al. (1998). Os resultados foram comparados em tabela contendo as seguintes informações: autores, objetivos específicos, amostra, desenho do estudo e principais resultados. Esta bibliografia comentada não identificou programas específicos de retorno ao trabalho para indivíduos com desordens musculoesqueléticas em MS, mas aponta para a multiplicidade de aspectos e atores envolvidos no processo, sendo necessária a realização de novos estudos.
\end{abstract}

DESCRITORES: Trabalho. Acidentes trabalho. Reabilitação vocacional.

\footnotetext{
* Trabalho apresentado como monografia para o curso de Especialização de Terapia Ocupacional - 2005 - UFMG.

${ }^{1}$ Terapeuta Ocupacional.

2 Terapeuta Ocupacional.

${ }^{3}$ Professora Assistente, Universidade Federal de Minas Gerais, Departamento de Terapia Ocupacional , Av. Antônio Carlos, 6627 - BH, MG. CEO: 31270-901. avaladao@ reitoria.ufmg.br

Endereço para correspondência: Sandra Ribeiro da Silva. Rua Ibatiba, 70 - Serrano - BH, MG, CEP: 30882-530. e-mail: sandra.to@hotmail.com
} 


\section{INTRODUÇÃO}

$\mathrm{O}$ mundo, nos últimos anos, tem sofrido mudanças na estrutura do trabalho. As indústrias modernizaram-se introduzindo novas tecnologias, acelerando o ritmo do trabalho, informatizando os serviços. Também surgiram novas profissões, ocorreu a globalização e as relações entre capital e trabalho foram redefinidas (FILHO, 1999; LANCMAN, 2004).

Esse cenário, segundo Lancman (2004), gerou um enorme impacto nas relações trabalho-emprego: surgiram novas formas de inserção no mercado de trabalho; ocorreu a redução de trabalho formal; migração da mão de obra das indústrias para o setor de serviços; o desemprego, o subemprego e o trabalho informal tornaram-se permanentes e estruturais.

Com o desemprego, subemprego e o aumento do trabalho informal, observou-se também a diminuição das garantias trabalhistas e previdenciárias, assim como o favorecimento de relações de trabalho precárias, o aumento da carga de tarefas para trabalhadores formais e a flexibilização de direitos trabalhistas (LANCMAN, 2004). Segundo a Organização Internacional do Trabalho (OIT, 2005), entre os anos de 1990 e 2003, na América Latina o aumento do número de empregos ocorreu principalmente no setor informal. De cada 10 novas pessoas ocupadas 6 estão no setor informal. Ainda conforme a OIT, houve uma redução na taxa de desemprego que, na América Latina e no Caribe, em 2004 chegou a $10,5 \%$, que equivale a 19,5 milhões de trabalhadores urbanos desempregados. Esta taxa foi ligeiramente inferior à observada em 2003 (11,1\%). No entanto, esta diminuição não contribuiu para uma melhoria significativa da situação do trabalho nestes países (OIT, 2004).

Paralelamente a esta mudança na estrutura do trabalho, nota-se um grande número de trabalhadores acometidos por doenças relacionadas ao trabalho ou por acidentes de trabalho, que resultam, muitas vezes, em incapacidades / doenças permanentes.

Estudos realizados em vários países têm demonstrado que trabalhadores do setor informal, temporários e terceirizados, estão envolvidos em atividades mais perigosas do que os trabalhadores formais, por vezes realizadas sem a proteção correta, colocando-os em maior risco de acidentes (SANTANAet al., 2003; FIGUEIREDO, 2004).

No entanto, segundo a OIT (2005) observa-se um declínio do número de acidentes de trabalho permanentes e temporários em todo o mundo, conforme demonstrado na Tabela 1. Uma das possíveis causas deste declínio é o aumento do emprego informal no mundo e, conseqüen- temente, a dificuldade em registrar acidentes de trabalho nesta população (GLINA; FELIPONE, 2004).

Tabela 1 - Número de acidentes permanentes/temporários em alguns países associados a OIT, que possuem registro de 2002 e 2003.

\begin{tabular}{l|l|l}
\hline Nome do País & $\mathbf{N}^{\mathbf{0}} /$ ano 2002 & $\mathbf{N}^{\mathbf{0}} /$ ano 2003 \\
\hline Austrália & 118.221 & 102.96 \\
Bulgária & 5.436 & 4.762 \\
Canadá & 359.174 & 348.854 \\
Costa Rica & 110.711 & 103.321 \\
Espanha & 935.198 & 898.983 \\
Finlândia & 57.767 & 56.268 \\
Hong Kong - China & 46.813 & 41.851 \\
Israel & 70.025 & 61.539 \\
Itália & 593.155 & 545.446 \\
Japão & 124.702 & 124.455 \\
México & 400.432 & 374.613 \\
Nova Zelândia & 24.249 & 23.778 \\
Suíça & 88.703 & 85.059 \\
Inglaterra & 159.608 & 164.767 \\
\hline
\end{tabular}

Fonte: Organização Internacional do Trabalho, 2005.

No Brasil (2005), segundo estatísticas do Anuário do Instituto Nacional de Seguro Social -INSS/Dataprev, no ano de 2003, o número de acidentes de trabalho e doenças ocupacionais foi de 390.180 representando uma queda de menos de $1 \%$ se comparado ao ano de 2002, como mostra a Tabela 2. Contudo, ressalta-se que esta queda também pode ser decorrente da sonegação de comunicação de acidentes de trabalho (CAT), do grande número de trabalhadores informais que não são cobertos pelo Seguro de Acidentes de Trabalho (SAT), e dos diversos regimes trabalhistas que não asseguram a notificação de doenças / acidentes de trabalho ao INSS, entre outros (LANCMAN, 2004).

Tabela 2 - Acidentes de trabalho e doenças ocupacionais no período entre 1999 a 2003 no Brasil.

\begin{tabular}{llllll}
\hline Ano & $\mathbf{1 9 9 9}$ & $\mathbf{2 0 0 0}$ & $\mathbf{2 0 0 1}$ & $\mathbf{2 0 0 2}$ & $\mathbf{2 0 0 3}$ \\
\hline Total/Brasil & 387.820 & 363.868 & 340.251 & 393.071 & 390.180 \\
\hline
\end{tabular}

Fonte: Brasil. Ministério da Previdência Social. Instituto Nacional de Seguridade Social - INSS.

Ainda com relação ao contingente de trabalhadores acometidos por doenças e acidentes do trabalho no Brasil, chamam a atenção a idade do trabalhador e a região do corpo acometida. De acordo com os dados do INSS (BRASIL, 2005), nota-se que os acidentes de trabalho ocorrem principalmente em adultos jovens, do sexo masculino, e em idade produtiva na faixa etária de 25 a 49 anos. 
As estatísticas do INSS (BRASIL, 2005) relatam que em $41,1 \%$ dos acidentes de trabalho registrados no Brasil em 2003, a região do corpo mais acometida foi o membro superior (MS) e que acidentes até o nível do punho representam $34,2 \%$ do total de acidentes do MS (BRASIL, 2004).

O membro superior, especialmente a mão, é de uso obrigatório para a realização da grande maioria das atividades da vida diária, podendo realizar forças de preensão de $45 \mathrm{~kg}$, bem como segurar e manipular linhas delicadas. É ainda capaz de empurrar, desferir golpes assim como ajudar na locomoção através de muletas e cadeiras de rodas (LEHEMKULL; SMITH, 1989). Segundo Lopes (2000), o valor real da mãoé muito grande, e talvez como a visão, é o órgão que maior número de conhecimentos transmite ao cérebro e que são guardados na memória. Sendo a mão um instrumento essencial para o desenvolvimento da tarefa de trabalho, qualquer acidente nesta região freqüentemente obriga o trabalhador a ausentar-se do trabalho, aumentando a incidência de afastamento nas estatísticas do INSS (LOPES, 2000). Se por um lado o afastamento causará impacto negativo para as empresas no que se refere aos custos com o trabalhador e à impossibilidade de nova contratação, por outro lado este impacto será muito maior para o indivíduo no que diz respeito ao desempenho de seu papel e função na vida cotidiana. Numa sociedade em que a identidade e valor pessoal estão estreitamente ligados ao papel de cada pessoa como profissional competitivo, retornar ao trabalho após o seu afastamento por doença ou acidente de trabalho se torna essencial (BURT, 2005). Neste sentido, o processo de reabilitação é fundamental.

Segundo Burt (2005), reabilitação significa o retorno à habilidade, ou seja, a volta às habilidades física, mental, social, vocacional e econômica da maneira mais completa possível para o indivíduo. Assim, o processo de reabilitação tem um papel primordial no retorno do trabalhador acidentado à suas funções/papéis e, paralelamente, na redução dos custos e perdas para o empregador causado pelo afastamento.

Embora haja consenso na literatura sobre a necessidade do processo de reabilitação, vários são os aspectos que podem ser importantes para a análise e o sucesso do mesmo. Atitudes pessoais e regras culturais são alguns exemplos. $\mathrm{O}$ valor do trabalho em uma determinada cultura pode ser altamente relevante para motivar o retorno ao mercado de trabalho. Além disso, apoio familiar, tipo de lesão, tempo de afastamento, processo de reabilitação, relação empregadoempregador, relação entre colegas de trabalho, políticas trabalhistas e de saúde podem diferenciar o processo de retorno ao trabalho em países de cultura, desenvolvimento e políticas públicas sociais diferentes (SIM, 1999; BURT, 2005).

A Commission On Accreditation Facilities - CARF
(Comissão de Credenciamento das Instalações de Reabilitação) (BURT, 2005) na legislação americana, definiu a preparação para o retorno ao trabalho como um programa de tratamento altamente estruturado e individualizado orientado a maximizar a capacidade de uma pessoa para retornar ao trabalho.

No Brasil, a saúde do trabalhador está regulamentada pela Lei Orgânica de Saúde, n ${ }^{\circ}$ 8.080, de 19 de setembro de 1990 (GLINA; FELIPONE, 2004). Esta lei, no entanto, não entra em detalhes sobre o que se espera sobre um processo de reabilitação para o retorno ao trabalho.

Diante deste panorama, onde se observam mudanças na organização do trabalho, o número crescente de trabalhadores com lesões nos MS, o alto custo pessoal e familiar gerado pela mudança nas funções/papéis dos indivíduos, o alto custo na manutenção dos trabalhadores lesados tanto para empresas quanto para governos, é importante que se invista em programas efetivos para o retorno do indivíduo ao trabalho. Portanto este estudo tem como objetivo realizar, por meio da bibliografia comentada, a análise criteriosa de artigos científicos que relatem aspectos de programas de retorno ao trabalho para indivíduos com desordens musculoesqueléticas nos MS identificando questões que influenciem na sua efetividade.

\section{METODOLOGIA}

Para este estudo foi realizada uma análise criteriosa de artigos científicos que se referiam ao retorno ao trabalho de pacientes com disfunções musculoesqueléticas em membros superiores, comparando-os através de uma bibliografia comentada.

A pesquisa foi realizada através de consultas em bibliotecas e nas bases de dados eletrônicas SCIELO, LILACS, PUBMED, MEDLINE, OTSEEKER, PEDRO e COCHRANE, seguindo como estratégia de busca as línguas inglesa e portuguesa, com investigação restrita ao período de 2000 até 2005 . As palavras-chave utilizadas foram: retorno trabalho em combinação com acidentes trabalho e saúde trabalhador e return work, returning work e vocational rehabilitation.

A seleção dos artigos foi feita por duas pesquisadoras. A seleção preliminar teve como critérios de inclusão: (1) existência, no título do artigo, das palavras return work, retorno trabalho e referência a desordens musculoesqueléticas; (2) período de publicação entre os anos de 2000 e 2005. Nova análise foi realizada a partir da leitura dos abstracts para selecionar aqueles que mencionavam membro superior.

Os artigos selecionados foram analisados criticamente, segundo a metodologia proposta por Law et al. (1998). Os 
estudos incluídos nesta revisão foram resumidos de forma padronizada, avaliando a citação completa do artigo, questão clínica de interesse, objetivo do estudo, literatura, delineamento do mesmo, amostragem, medidas, intervenção, resultados, conclusões e implicações clínicas.

\section{RESULTADOS}

Nesta pesquisa foi identificado um total de 9810 artigos (MEDLINE - 3123, PUBMED - 5186, LILACS - 12, COCHRANE-971,PEDRO-159, OTSEEKER-359), dos quais 87 foram selecionados por se adequarem aos critérios de inclusão. Após a leitura dos abstracts foram selecionados 13 artigos e excluídos 74 . Considerando que quatro destes artigos estavam disponíveis somente por pedido via correio e, tendo em vista o tempo previsto para a realização deste estudo, optou-se por utilizar os nove artigos disponibilizados no portal CAPES.

Os nove artigos selecionados foram resumidos conforme a análise crítica sugerida por Law et al. (1998), com as seguintes informações: autores, objetivos específicos, amostra, desenho do estudo e principais resultados, como mostra a Tabela 3.

Dentre os artigos selecionados dois são do ano de 2005, um de 2004, quatro de 2003 e dois de 2002, sendo 2 revisões sistemáticas e 7 estudos quantitativos. Em comum os artigos apresentam a preocupação com o retorno ao trabalho o mais precocemente possível, mas investigam pontos distintos como o tempo que trabalhadores com ou sem seguro levam para retornar ao trabalho; a efetividade dos tratamentos para o retorno ao trabalho; as influências das características individuais dos trabalhadores para o retorno ao trabalho.

\section{DISCUSSÃO}

Os artigos avaliados são voltados para o estudo de indivíduos com lesões por acidentes/doenças dos MS relacionadas ao trabalho e apontam para a multiplicidade de fatores envolvidos no processo de retorno ao trabalho após doença ou acidente. Destacam-se questões psicológicas, físicas, nível educacional, reabilitação física, tipos e local da lesão, tempo de afastamento, tipo de seguro do trabalhador ou a sua falta, possibilidade de trabalho modificado, relações com a empresa e colegas de trabalho, políticas organizacionais e práticas das empresas.

O aumento, nos últimos anos, de desordens musculoesqueléticas que afetam o MS foi ressaltado por muitos autores (ADAMS; WILLIAMS, 2003; BRUYNS et al., 2003; MEIJER et al., 2005) e MEIJER et al. (2005) acrescentam que as desordens musculoesqueléticas de MS podem ser agudas e autolimitantes e ocasionalmente crônicas e persistentes.

A complexidade das lesões de MS dificulta o retorno ao trabalho dos indivíduos, sendo que a dor aparece como importante fator no processo de retorno ao trabalho conforme relatam Ferestein et al. (2003), Adams e Willians (2003) e Selander et al. (2002), podendo dificultar e /ou retardar o retorno ao trabalho.

Lesões de nervos periféricos foram encontradas em 5 dos textos revisados (BITAR et al., 2002; BRUYNS et al., 2003; FEUERSTEIN et al., 2003; CHAISE et al., 2004; OSSMAN et al., 2005), sendo a síndrome do túnel do carpo considerada como um desafio entre as desordens musculoesqueléticas de MS. Feuerstein el al. (2003)destacam que indivíduos com diagnósticos de mononeuropatias informam mais dor, menos função, necessidade de maior número de cirurgias e menor taxa de retorno ao trabalho. Bruyns (2003) aponta que há diferença significante para o retorno ao trabalho de pacientes com lesão concomitante de nervo mediano e nervo ulnar em relação a pacientes com lesão isolada de um destes nervos $\mathrm{E}$ nas lesões isoladas o percentual de retorno ao trabalho dos indivíduos com lesão de nervo mediano é maior do que o dos indivíduos com lesão de nervo ulnar.

Como evidencia a literatura o afastamento do trabalho acarreta perdas em todos os sentidos: pessoais, familiares, sociais e econômicas. Desta forma, é importante, tanto para os indivíduos como para a sociedade, retornar os trabalhadores ao ambiente de trabalho. Contudo, o retorno ao trabalho pode depender e ser influenciado por fatores psicológicos, sociais, médicos, reabilitação, local de trabalho, sistemas de benefícios, idade e grau de escolaridade, conforme descreve Selander et al. (2002).

Fatores físicos, nível educacional e o envolvimento do trabalhador foram apontados também em outros textos como influenciadores para o retorno ao trabalho (ADAMS; WILLIANS, 2003; MEIJER et al., 2005). Rusch et al. (2003) ressaltam que os trabalhadores que se consideram responsáveis por seu acidente são menos resistentes em retornar as suas atividades de trabalho anteriores do que aqueles que culparam o colega de trabalho ou o equipamento por suas lesões.

Ao se considerar o tempo de permanência fora do local de trabalho, pode-se verificar que quanto maior o período da licença médica, mais difícil é o retorno do trabalhador ao emprego (MEIJER et al., 2005). Levando-se em conta a diferença entre licenças médicas e de tipos de serviços Bitar et al. (2005) avaliaram que um grupo dos EUA com seguro apresentavam um tempo de retorno ao trabalho mais prolongado do que se comparado a outro grupo dos EUA 
sem seguro ou a um grupo da Suécia. Já Chaisse (2004) evidencia em suas pesquisas que trabalhadores do serviço público tiveram um intervalo de tempo fora do trabalho maior que os do setor privado e que os independentes. Este mesmo autor mostra que o seguro social têm influencia no tempo de retorno ao trabalho após cirurgia de síndrome do túnel do carpo, sendo que os trabalhadores com indenização apresentam um intervalo maior de retorno ao trabalho do que trabalhadores com seguro social.

Sabe-se que o afastamento do trabalho, além de gerar custos pessoais e familiares para o indivíduo, acarreta também gastos para as empresas e governos com a impossibilidade de nova contratação, a manutenção do tratamento e benefícios. Uma maneira de diminuir este período de afastamento é o trabalho modificado. Ferestein et al. (2003) descrevem em seu estudo que, apesar do desconforto prolongado e das limitações funcionais, 1/3 dos trabalhadores com desordens musculoesqueléticas em MS não eram capazes de retornar ao trabalho normal, mas podiam executar um trabalho modificado. Com este estudo eles apontam para a possibilidade de retorno ao trabalho modificado quando se está diante de limitações significativas e ressaltam a escassez de estudos sobre trabalho modificado e desordens musculoesqueléticas.

Quando se pensa em retornar um indivíduo ao seu ambiente de trabalho, além da reabilitação física e psicológica, do suporte familiar e social, também é importante a participação das empresas e o apoio dos colegas de trabalho.

Ossmann et al. (2005) propuseram avaliar o acordo entre administração e empregados e as políticas organizacionais e práticas (OPP) envolvidas no processo de retorno ao trabalho após cirurgia do túnel do carpo. Os resultados preliminares sugerem que as avaliações de empregados são preditivas de incidência de doenças, de inaptidão para o trabalho, retorno ao trabalho e apoio organizacional.E que empresas maiores oferecem melhores estruturas práticas e políticas relacionadas à saúde dos trabalhadores.

Dentre os artigos revisados não foi possível verificar nenhum programa específico de retorno ao trabalho para indivíduos com lesões musculoesqueléticas do MS, apesar de se constatar a necessidade do retorno ao trabalho após doença ou lesão em MS ou em outras regiões do corpo.

\section{CONCLUSÃO}

Os resultados desta bibliografia comentada evidenciam a necessidade e a preocupação em retornar ao trabalho o mais precocemente possível, evitando o desgaste emocional e perdas pessoais para o trabalhador, bem como os custos gerados para o empregador e governos.

Os aspectos psicológicos e individuais têm grande relevância quanto ao retorno ao trabalho, como também fatores sociais, econômicos, médicos, reabilitação, local de trabalho, sistemas de beneficio e grau de escolaridade. Outros aspectos que podem influenciar o retorno ao trabalho de indivíduos que sofreram lesões em MS são o nível de dor e o tipo de lesão.

Sabendo-se dos fatores relevantes que podem influenciar no retorno ao trabalho bem sucedido e da importância deste retorno para os trabalhadores, torna-se necessária a criação de programas efetivos que possam ser utilizados nesta população e que possibilitem o sucesso do retorno ao trabalho.

$\overline{\text { SILVA, S. R.; GUIMARÃES, E. V.; RODRIGUES, A. M. V. N. Aspects connected with retorning to work }}$ process of individuals with musculoesquelectical desorders: a commented bibliography. Rev. Ter. Ocup. Univ. São Paulo, v. 18, n. 1, p. 38-43, jan./abr., 2007.

ABSTRACT: Return to the work after an accident / illness is presented as a constant challenge for individuals, governments, and professionals of rehabilitation, becoming reason of constant concerns of researchers. Current researches have detached the increase of upper extremity musculoskeletal disorders (MS) in the whole world. In Brazil, according to statisticians of National Institute of Social Security (INSS), 41,1\% of the accidents registered in 2003 were in MS. This work had the objective to accomplish, through a commented on bibliography, the detailed scientific article analysis reporting the several aspects of the return to work programs for individuals with upper extremity musculoskeletal disorders, identifying factors to influence in their effectiveness. The electronic database between 2000 and 2005 was consulted. According inclusion criteria, nine articles were selected and analyzed the quality of the evidences as the methodology proposed by Law et al. (1998). The results were compared in table containing the following information: authors, specific objectives, sample, drawing of the study and main results. This commented on bibliography didn't identify specific programs of return to work for individuals with upper extremity musculoskeletal disorders, but it appears for the multiplicity of aspects and actors involved in the process.

KEY WORDS: Work. Accidents, occupational. Rehabilitation, vocational. 


\section{REFERÊNCIAS}

ADAMS, J. H.; WILLIANS, A. C. C. What affects return to work for graduates of a pain management program with chronic upper limb pain? J. Occup. Rehabil., v. 13, n. 2, p. 91-105, 2003.

BITAR, G. et al. Carpal tunnel release in the United States and Sweden: Reimbursement patterns, cost for treatment, and return to work. Plastic Reconstr. Surg., v. 109, n. 5, p. 1574-80, 2002.

BRASIL. Ministério da Previdência Social. Instituto Nacional de Seguridade Social. Estatísticas sobre acidentes de trabalho. Disponível em: http://www.inss.gov.br. Acesso em 16/07/2005.

BRASIL. Ministério do Trabalho e Emprego. Estatísticas sobre acidentes de trabalho, 2004. Disponível em: http:// www.mte.gov.br. Acesso em: 01/09/2005.

BRUYNS, C. N. P. et al. Predictors for return to work in patients with median and ulnar nerve injuries. J. Hand Surg., v. 28, n. 1, p. 28-33, 2003

BURT, C. M. Avaliação do trabalho e preparação para o retorno ao trabalho. In: PEDRETTI, L. W.; EARLY, M. B. Terapia ocupacional: capacidades práticas para disfunções físicas. 2005. p. 241-254.

CHAISE, F.; BELLEMERE, P.; FRIL, J. P.; GAISNE, E.; POIRIER, P.; MENADI A. Return to work internal and surgery for carpal tunnel syndrome. results of a prospective series of 233 patients. J. Hand Surg., v. 298, n. 6, p. 568-570, 2004.

FEUERSTEIN, M.; SHAW, W. S.; LINCOLN, A. E.; MILLER, V. I.; WOOD, P. M. Clinical and workplace factors associated with a return to modified duty in work-related upper extremity disorders. Pain, v. 102, p. 51-61, 2003.

FIGUEIREDO, I. M. Lesões de mão no trabalho: análise dos casos atentidos do Serviço de Terapia Ocupacional de um hospital público de Belo Horizonte - MG. 2004. Dissertação (Mestrado Ciências de Reabilitação) - Universidade Federal de Minas Gerais, Belo Horizonte, 2004.

FILHO, V. W. Reestruturação produtiva e acidentes de trabalho no Brasil: estrutura e tendências. Cad. Saúde Pública, v. 15, n. 1, p. 41-51, 1999.

GLINA, D. M. R.; FELIPONE, S. M. N. et al. Grupo de reorientação profissional com trabalhadores: nova proposta na saúde do trabalhador? In: Saúde, trabalho e terapia ocupacional. São Paulo: Roca, 2004.

LANCMAN, S. Construção de novas teorias e práticas em terapia ocupacional, saúde e trabalho. In: LANCMAN, S. Saúde, trabalho e terapia ocupacional. São Paulo: Roca, 2004.

LAW, M. et al. The critically appraised topic - CAT - McMaster University - Canadá) Critical review from quantitative studies [1998]. Disponível em: http://www.fhs.mcmaster.ca/rehab/ebp. Acesso em 20 ago. 2005.

LEHEMKULL, L. D.; SMITH, L. Cinesiologia clínica de Brunnstrom. $4^{\text {a }}$ ed. São Paulo: Manole, 1989.

LOPES, E. I. Aspectos sociais e econômicos dos traumatismos da Mão In: PARDINI, A. G. Traumatismos da mão. 3a ed. São Paulo: Medsi, 2000.

MEIJER, E. M. et al. Evaluation of effective return to work treatment programs for sick-listed patients with non-specific musculoskeletal complaints: a systematic review. Int. Arch. Occup. Environ. Health, v. 78, p. 523-532, 2005.

OFICINA INTERNACIONAL DEL TRABAJO - OIT. Panorama laboral - América Latina e Caribe. 2004. Disponível em: http://www.oitbrasil.org.br/info/estat.php. Acesso em 25/08/ 2005.

ORGANIZAÇÃO INTERNACIIONAL DO TRABALHO OIT. Cases of injury with lost workdays, by economic activity, 2005. Disponível em: www.mte.gov.br . Acesso em: 11/09/2005.

OSSMAN, J. et al. Management and employee agreement on reports of organizational policies and practices important in return to work following carpal tunnel surgery. J. Occup. Rehabil., v. 15, n. 1, p. 17-26, 2005.

RUSCH, M.; DZWIERZYNSKI, W. W.; SANGER, J. R.; PRUIT, N. T.; SIEWERT, A. D. Return to work outcomes after work related hand trauma: the role of causal attributions. J. Hand Surg., v. 28A, n. 4, p.673-677, 2003.

SANTANA, V.; MARNETOFT, S. V.; BERGROTH, A.; EKHOLM, J. Acidentes de trabalho não fatais: diferenças de gênero e tipo de contrato de trabalho. Cad. Saúde Pública, v. 19, n. 2, p. 481-493, 2003.

SELANDER, J. et al. Return to work following vocational rehabilitation for neck, back and shoulder problems: risk factors reviewed. Disabil. Rehabil., v. 24, n. 14, p.704-712, 2002.

SIM, J. Improving Return-to-work strategies in the United States disability programs, with analysis of program practices in Germany and Sweden. Social Security Bull., v.62, n. 3, p. 41-49, 1999. 